\title{
Channel encoding system for transmitting image over wireless network
}

\author{
Bharathi Gururaj $^{1}$, G. Sadashivappa ${ }^{2}$ \\ ${ }^{1}$ Department of Electronics and Communication Engineering, ACS College of Engineering, India \\ ${ }^{2}$ Department of Telecommunication Engineering, R V College of Engineering, India
}

\begin{tabular}{l}
\hline \hline Article Info \\
\hline Article history: \\
Received Aug 19, 2019 \\
Revised Mar 3, 2020 \\
Accepted Mar 21, 2020 \\
\hline Keywords: \\
Channel encoding \\
Error-free transmission \\
Image transmission \\
Lossless \\
Signal quality \\
Wireless communication \\
\hline
\end{tabular}

\begin{abstract}
Various encoding schemes have been introduced till date focusing on an effective image transmission scheme in presence of error-prone artifacts in wireless communication channel. Review of existing schemes of channel encoding systems infer that they are mostly inclined on compression scheme and less over problems of superior retention of signal retention as they lacks an essential consideration of network states. Therefore, the proposed manuscript introduces a cost effective lossless encoding scheme which ensures resilient transmission of different forms of images. Adopting an analytical research methodology, the modeling has been carried out to ensure that a novel series of encoding operation be performed over an image followed by an effective indexing mechanism. The study outcome confirms that proposed system outshines existing encoding schemes in every respect.
\end{abstract}

Copyright $(0) 2020$ Institute of Advanced Engineering and Science. All rights reserved.

\section{Corresponding Author:}

Bharathi Gururaj,

Department of Electronics and Communication Engineering,

ACS College of Engineering,

Mysore Road, Bengaluru, Karnataka 560074, India.

Email: bharathigururaj@gmail.com

\section{INTRODUCTION}

Wireless communication system is one of the most frequently adopted communication system in the present era owing to its supportive to various technologies as well as cost effectiveness [1, 2]. However, it is also shrouded with various challenges e.g. fading, scattering, interference, noise, etc. [3]. Irrespective of various studies towards addressing an effective wireless communication system, the problem of quality transmission of the data file from transmitting point to receiving point is yet an open-end problem [4-6]. The biggest impact of the adverse state of wireless communication system is affected when multimedia transmission is carried out [7]. Usually, multimedia file systems are bigger sized digital content that are transmitted over any network. There are various schemes which address multimedia transmission as well as streaming over wireless channel [8]. In this regard, image is one of the frequently used digital content adopted by wide range of user's right from social network to healthcare sector. Different forms of encoding schemes have been evolved in due course of time for facilitating an effective transmission of an image [9]; however, majority of the existing encoding schemes are inflicted with certain range of problems. If these problems are not solved than it will degrade quality of experience for many of the application where accuracy is of utmost priority e.g. healthcare sector, defense sector, meteorological sector, etc. Hence, there is a need of an effective modeling mechanism that emphasize over incorporating network related parameters in channel encoding system for ensuring robust image transmission over wireless network. Therefore, this paper introduces a simplified model that is capable of performing a comprehensive encoding mechanism for ensuring error resistive transmission scheme over wireless channel. Section 2 discusses about proposed methodology followed by elaborated discussion of algorithm implementation in Section 3. Comparative analysis of accomplished result is discussed under Section 4 followed by conclusion in Section 5 . 
There are various approaches where different variants of encoding systems have been introduced in past [10]. Most recently, the work carried out by Mhamdi et al. [11] have used turbo coding system for enhancing the wireless transmission of image encoded by JPEG2000 standard. Low-Density Parity Check (LDPC) is another frequently used approach for encoding image and claimed to offer better optimized performance as seen in the work of Deng et al. [12] and Bocharova et al. [13]. The work of Matin et al. [14] has applied optical encoding system along with the potential of compressive sensing for better optimized results of image transmission. Existing system has also witnessed usage of Hadamard coding system over medical images as evident in work of Shen and Yan [15]. Adoption of enhanced LDPC codes has been seen in model of He et al. [16] where the authors have uses approximation approach. The work carried out by Gong et al. [17-20] have used similar Hadamard codes over medical imaging system for facilitating better encoding system with good support over delay-based transmission system. A different usage of Hadamard codes have been mechanized in the work of Liu and Luo [21] where compressive sensing has been used for image transmission over wireless networks. The work of Villaverde et al. [22] has also used Hadamard codes for assisting in non-destructive evaluation of signal. Adoption of compressive sensing using distributed approach is seen in work of Song et al. [23] targeting for transmission of image over wireless medium. Existing system has also used Markov field using LDPC for performing compression of encrypted image as seen in work of Wang et al. [24]. Utilization of the compressive sensing along with rate distortion concept has been carried out by Chen et al. [25] where scrambled block has been used for transmission of image. Optical encoding system is another frequently used approach tested over photonic imaging system as witnessed in work of Chen [26]. Apart from this there are various other approaches e.g. addressing structurization of block using LDPC [27], turbo coding over multiple-folds [28], LDPC based compressive sensing [29], and lossless encoding over complex image form [30]. Hence, there are multiple variants of encoding approaches attempted over image transmission in existing system. Mahboub et al. [31] demonstartes a model called Hybrid SEP: The FMZ clustering protocol is done based on stable election model. The work of Shanthi [32] have introduced heuristic based energy efficient broadcasting method. The result show $\mathrm{s}$ that the stabily time of FMZ-SEP. Hassanet et al. [33] has presented estimation the performance of fuczzy logic algorithm and K-means in order to configuration balanced clusters in the WSNs. The next section discusses about research problem.

The significant research problem explored from existing literatures are as follows viz. i) majority of existing encoding mechanism emphasizes about compression performance and not actually about the signal quality of the reconstructed image, ii) existing encoding approaches doesn't emphasize much over the possibilities of dynamic artifacts over the wireless medium, iii) there are no much consideration of network related parameters found in the existing encoding approach. Apart from this, there is no much approach which has discussed about cost effective modeling using a novel encoding technique. Therefore, the research problem could be stated as "developing a comprehensive encoding mechanism that facilitates image transmission over dynamic wireless network with uncertain artifacts with assurance of optimal signal quality of reconstructed image."

\section{PROPOSED METHODOLOGY}

The proposed work is an extension of the prior work [34] where an image transmission method was discussed. This part of the current work further extends the model by emphasizing on state of communication channel and novel encoding system. The adopted scheme is showcased in Figure 1. The figure highlights the scheme used in the proposed system where the prime emphasis is given to the network-based encoding scheme. The study addresses the existing system by incorporating the problems associated with wireless communication and its possible effect over the quality of an image. The system also introduces a series of encoding operation which considers the compression ratio and make sure that there is no significant impact on its quality of reconstructed image irrespective of any traffic condition. The next section illustrates about the system design adopted for implementing the proposed encoding scheme.

\section{SYSTEM IMPLEMENTATION}

The complete implementation of the proposed system is carried out considering the transmission of an image through an error-prone communication channel. The prime agenda of the implementation design is to ensure that irrespective of any presence of artifacts, there should be highly reduced degradation of the quality of the received image. This section discusses about the strategies adopted for implementation of the proposed system followed by highlights of the process being involved in the proposed transmission mechanism with ensured quality of image. 


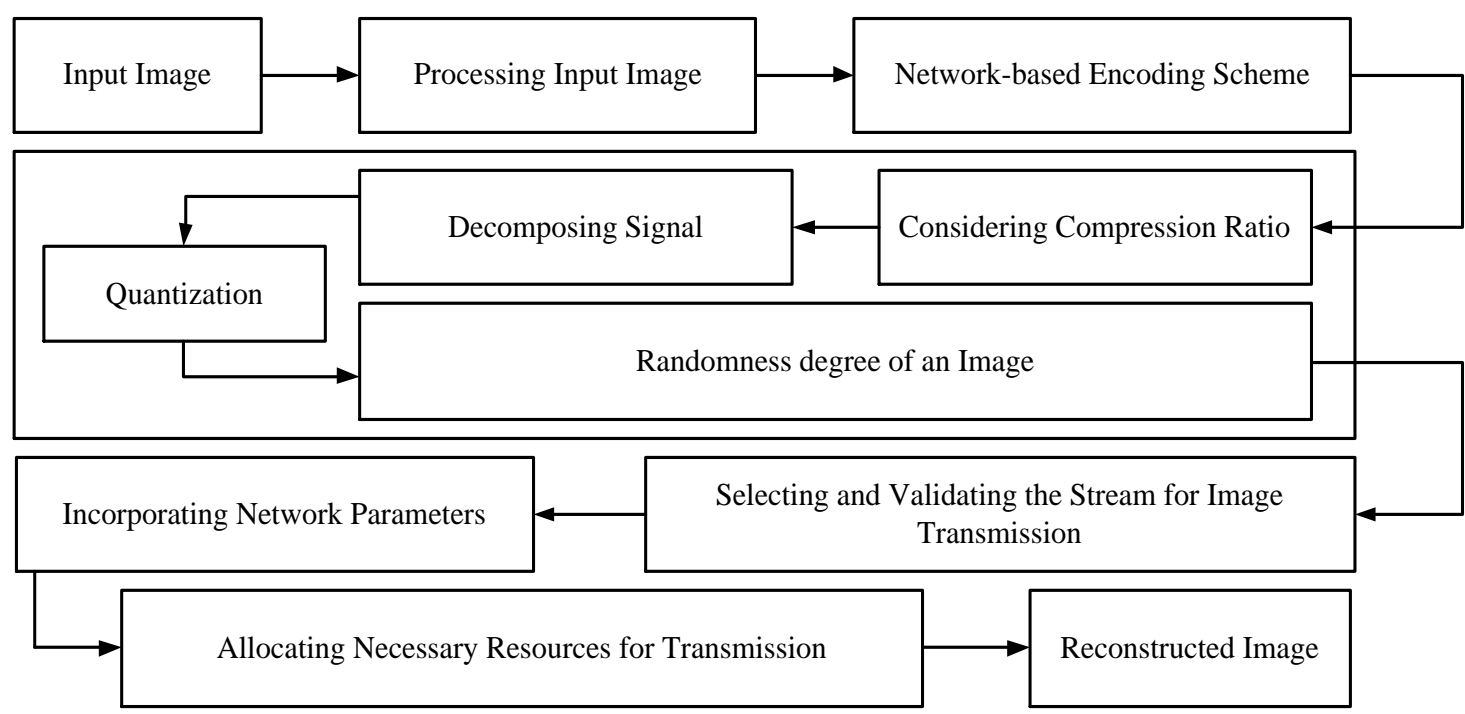

Figure 1. Considered scheme of methodology

\subsection{Strategies adopted for implementation}

The cycle of implementation of proposed system is carried out on the basis of the consideration that a specific communication channel to be designed followed by processing on the image to be transmitted. The primary strategy of proposed implementation is that a communication channel with frequency used encoding mechanism to be used in order to map with the practical transmission scenario. The secondary strategy of proposed system is to perform packetization of the image to be transmitted on the basis of the transmission load over the adopted communication channel. The tertiary strategy of the proposed system is to incorporate a proper selection of communication stream followed by assigning only demanded resources for assisting error-free transmission of image.

\subsection{Assumptions and dependencies}

As the proposed system targets to forward the image over the error-prone communication channel therefore it is necessary to assume that it offers wide range of supportability to various forms and types of an image. It will eventually mean that proposed system will offer uniformity in signal quality in the receiver end irrespective of the type of the image to be forwarded. The communication channel is assumed to possess artifacts originated from traffic load and not from other reasons e.g. security breach. It is also assumed that there exist various forms of network protocols each executing its own protocols over the image to be forwarded. Therefore, the core dependency of proposed system will be to consider a range of compression ratio for the purpose of investigating the impact of compression ratio over the signal quality. By incorporating such method, the proposed system will be feasible to offer the outcome as a result of various underlying network protocols running.

\subsection{Core process flow involved in implementation}

The core process flows see Figure 2 of the proposed system are as follows:

a. Processing input image: The first step of the proposed system is to take the input image to be forwarded. In order to support various forms and dimension of an image. Usually, the dimension of standard to high-definition images resides between 256x256 to 1024x1024 pixels. Therefore, irrespective of the size of an image, the proposed system is capable of process any form as well as dimensions of the image to be forwarded.

b. Network-based encoding scheme: The proposed system makes use of embedded block coding system which assists in the significant control over the latency in presence of the communication channel with an artifact. For this purpose, the proposed system carry out series of associated operation:

- Considering compression ratio: The proposed system can consider multiple range of compression ratio in order to assess the impact of it over the signal quality. The logic of compression ratio is that-more traffic load will lead lower the rate of packet transmission which will lead to perform frequent compression operation. Therefore, considering compression ratio gives an edge to understand the impact of traffic load over the image transmission process. 
- Decomposing signal: In order to ensure that proposed system could forward the bigger packet of an image of large dimension over a congested network, it is necessary to ensure that they can be forwarded without affecting resolution. For this purpose, the proposed system considers decomposing the image signals with multiple precision levels over a multiple-level of decomposition. This operation will yield a process where a bigger image can be splitted into smaller version of it that can be easily forwarded to the congested traffic condition too. The degree of congestion can be controlled by configuring the value of compression ratio. Higher traffic load will mean lower capability of compression and vice-versa.

- Quantization: The prior step of signal decomposition will lead to generation of multiple numbers of decomposed signals which could be further reduced. For this purpose, the system considers a unit value of quantum which is considered as a reference point that is used for considering ranges of pixels for performing compression. This operation will lead to generation of reduced size of the image to be transmitted as an extra step of optimization. The quantization operation is carried out over multiple decomposed coefficient of the image offering an advantage towards overhead as well as data transmission over peak traffic condition.

Randomness degree of an image: After the quantization is performed, it can be said that significant lossless encoding mechanism has been incorporated. However, the size of the coding length was overlooked in the prior step. Therefore, this step computes the randomness degree of an image in order to reduce the range of the mean length of the coding with respect to bits per pixel. This operation assists in better form of practical realization along with assurance of zero information being lost in the communication channel with peak traffic condition. At the same time, the complete process can control the scale of randomness on the basis of the application demands and hence it is quite user-friendly and effective against any form of communication channel.

c. Incorporating network parameters: As the proposed system deploys the concept of forwarding the image over the communication network therefore it is essential to consider the transmission related parameters. The proposed system considers presence of multiple numbers of streams where the image will be allocated to be forwarded from transmitter to receiver end. The study considers that there are $n$ number of finite streams (value of $\mathrm{n}$ depends upon the consideration of the communication system). An assignment of $m$ number of data packets of the input image is carried out per $n$ stream to make it more measurable mapping with the practical communication environment. For an effective transmission, the study considers that $m>n$.

$\mathrm{d}$. Selecting and validating the stream for image transmission: After taking the input of $\mathrm{m}$ and $\mathrm{n}$ from the prior step, the proposed system initializes the value for channel state parameter $C_{p}$, transmission rate $t_{r}$, and error-reduction coefficient ercoef. All the data packets are transformed in its smallest entity of bits. The system than allocates compressed its data to be forwarded as per the value of $\mathrm{m}$ and $\mathrm{n}$ respectively. A matrix is considered which repositsm and $\mathrm{n}$ in order to create an index of the communication link. After the bits of the data packets are obtained all the transposition operation is carried out over the bit streams followed by using a function capable of mapping the image data of any dimension into the fixed dimensional data. This process offers a uniform performance over the network irrespective of the size of the image as well as it is also free from any traffic load factor too. The indexed value of the bit streams are further maintained in matrix.

e. Allocating necessary resources for transmission: It is essential to compute the necessary network related resources prior to perform transmission of image to error-prone communication channel. For this purpose, an unique encoding system is introduced which extracts the index of the data obtained from prior stream and concatenates with the next bit stream of the communication link. As the complete route considered for the data communication is already indexed, therefore, all the information associated with the communication channel can be reposited. The advantage of this mechanism is that size of the forwarded data remains very small as the indexed data size is very small compared to the original data (i.e. image) which offers and advantage to easily forwarded to the destination node.

The next process is to transmit the encoded as well as indexed data and all the above mentioned operation is quite necessary prior to forwarding the image to destination node. The receiver node then obtains the data sequentially without any loss of data as complete encoded data is maintained in indexed form which can be easily validated. Finally, all the indexed data are decoded to its natural state of bits which can be directly accessed by the user. The significant advantage of the above mentioned series of processes is that proposed system offers potential reduction in the actual size of the data followed by allocation of the necessary resources as well as cross checking of the index files used in the process. Apart from this, the complete process of transmission becomes faster without any loss of information making the system cost effective. 


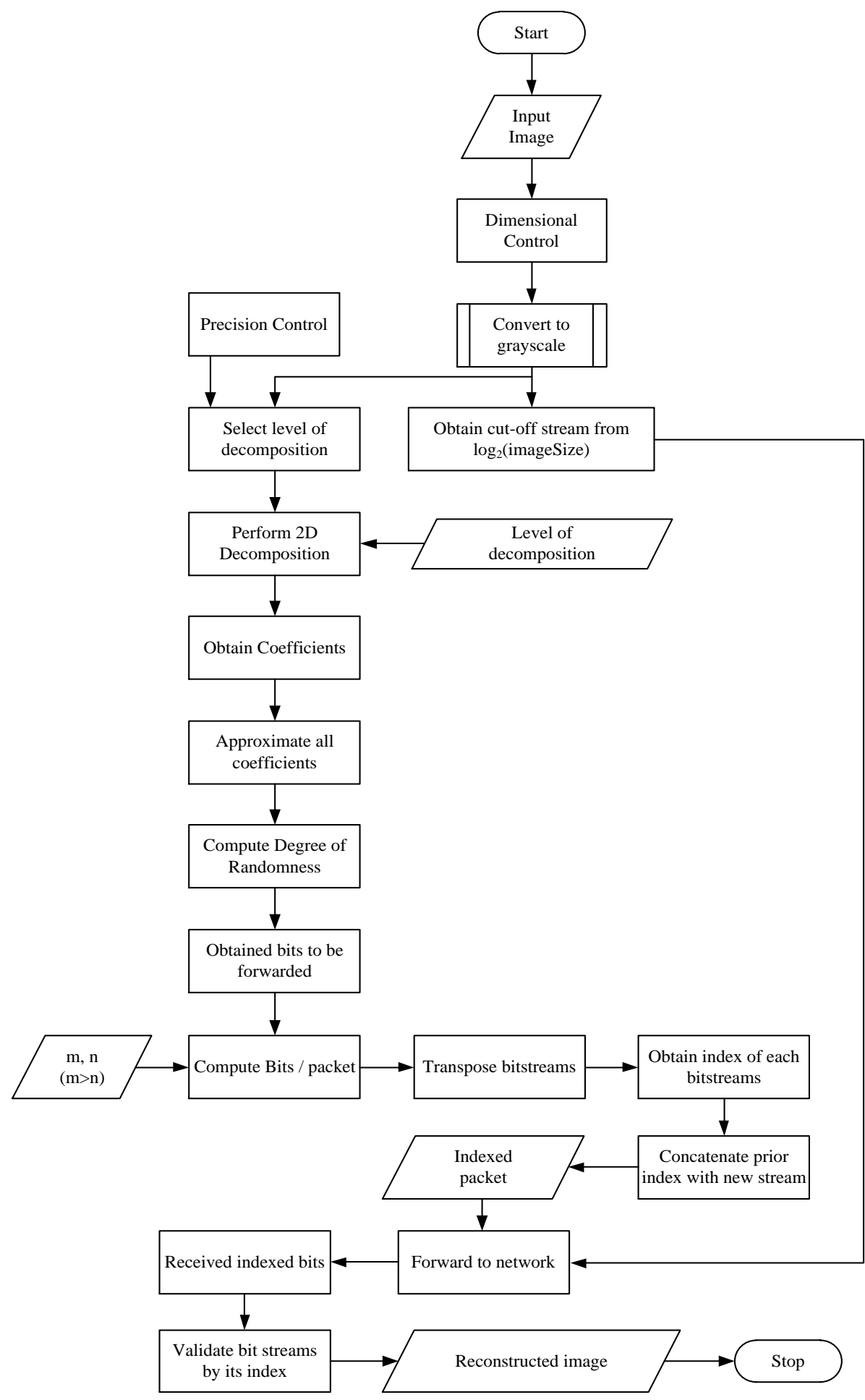

Figure 2. Process flow of the proposed system

\section{RESULTS DISCUSSION}

The scripting of the proposed logic was carried out in MATLAB considering multiple types of images with respect to size, color, dimension, standard, real-time, etc. The analysis is carried out considering a standard wireless network considering $\mathrm{C}_{\mathrm{p}}=0.2$ (dimensionless unit), $t_{\mathrm{r}}=10 \mathrm{kbps}$, and ercoef $=0.1$ (dimensionless unit). The number of bits to be forwarded is calculated by obtaining length of communication bits divided by product of $m$ and $n$. The sample visual outcome exhibited in Table 1 showcase that there is no significant differences of the encoding performance irrespective of the type of images. 
The study outcome is also compared with the existing frequently used channel encoding schemes e.g. convolution coding, turbo coding, LDPC coding. Table 2 highlights the numerical outcomes of proposed system and existing system obtained by averaging the numerical scores obtained by implementing individual existing coding system. The outcome evidently shows that proposed system offers significantly better signal quality as seen from good Peak Signal-to-Noise Ratio (PSNR), good resolution as seen from bits per pixel while better compression performance too. Apart from this, the overall processing time of proposed system is found to be 0.18772 seconds while that of existing encoding system is 2.65886 seconds processed over core-i3/5 processor. Hence, it can be said that proposed system offers practical encoding scheme for wireless transmission of image.

Table 1. Visual outcome of study

\begin{tabular}{|c|c|c|c|}
\hline & Input Image & Encoded Image & Reconstrcted Image \\
\hline $\begin{array}{c}\text { Standard Image } \\
256 \times 256\end{array}$ & & & \\
\hline $\begin{array}{l}\text { Real-time Image } \\
\quad 700 \times 700\end{array}$ & & & \\
\hline
\end{tabular}

Table 2. Numerical outcome of study

\begin{tabular}{ccccc}
\hline & \multicolumn{2}{c}{ Existing } & \multicolumn{2}{c}{ Proposed Enco } \\
& Standard & Real-time & Standard & Real-time \\
\hline PSNR & 16 & 27 & $25 \mathrm{~dB}$ & $37 \mathrm{~dB}$ \\
Bits per Pixel & 0.11 & 0.19 & $0.2 \mathrm{bpp}$ & $0.3 \mathrm{bpp}$ \\
Compression \% & $62 \%$ & $67 \%$ & $72 \%$ & $85 \%$ \\
\hline
\end{tabular}

\section{CONCLUSION}

In current age of digital communication, image is one of the easier mechanisms to transmit information. However, there is various form of application where certain application demands $100 \%$ integrity of the image. Such integrity of the image may be corrupted due to error-prone wireless transmission system and therefore a robust channel encoding system is demanded. The proposed system introduced a simplified model where a comprehensive channel encoding is carried out in order to an effective transmission. The contributions of the proposed system are as follows: i) the performance of the channel encoding of proposed system is consistent irrespective of various types/dimensions of the image. ii) The complete encoding scheme is carried out based on packetization concept where the communication bits are formulated based on the current traffic load to offer higher flexibility in transmission. iii) An effective indexing mechanism has been carried out in existing system, which significantly reduces the size of the transmitted image with absolutely no effect on the signal quality of the reconstructed image.

\section{REFERENCES}

[1] A. Mahboub, et al., "An energy-efficient clustering protocol using fuzzy logic and network segmentation for heterogeneous WSN," International Journal of Electrical and Computer Engineering (IJECE), vol. 9, no. 5, pp. 4192-4203, 2019.

[2] N. S. Ali, Z. A. A. Alyasseri, and A. Abdulmohson, "Real-Time Heart Pulse Monitoring Technique Using Wireless Sensor Network and Mobile Application," International Journal of Electrical and Computer Engineering (IJECE), vol. 8, no. 6, pp. 5118-5126, 2018. 
[3] A. T. Khalil, A. I. Abdel-Fatah, and H. A. Sakr, "Rapidly IPv6 multimedia management schemes based LTE-A wireless networks," International Journal of Electrical and Computer Engineering (IJECE), vol. 9, no. 4, pp. 3077-3089, 2019.

[4] G. Khanna and S. K. Chaturvedi, "A Comprehensive Survey on Multi-hop Wireless Networks: Milestones, Changing Trends and Concomitant Challenges," ACM-Journal Wireless Personal Communications: An International Journal, vol. 101, no. 2, 2018.

[5] F. Jameel, et al., "A Survey of Device-to-Device Communications: Research Issues and Challenges," IEEE Communications Surveys \& Tutorials, vol. 20, no. 3, pp. 2133-2168, 2018, doi: 10.1109/COMST.2018.2828120.

[6] X. B. Maxama and E. D. Markus, "A Survey on Propagation Challenges in Wireless Communication Networks over Irregular Terrains," in 2018 Open Innovations Conference (OI), Johannesburg, pp. 79-86, 2018.

[7] Z. Akhtar and T. H. Falk, "Audio-Visual Multimedia Quality Assessment: A Comprehensive Survey," IEEE Access, vol. 5, pp. 21090-21117, 2017

[8] M. Wang, et al., "Video streaming distribution over mobile Internet: a survey," Frontiers of Computer Science, vol. 12, no. 6, pp. 1039-1059, 2018

[9] A. Singh and K. G. Kirar, "Review of image compression techniques," in 2017 International Conference on Recent Innovations in Signal processing and Embedded Systems (RISE), Bhopal, pp. 172-174, 2017.

[10] B. Gururaj and G Sadashivappa, "Insights on Error-Resilient Image Transmission Schemes on Wireless Network," International Journal of Advanced Computer Science and Applications, vol. 8, no. 1, 2017.

[11] M. Mhamdi, et al., "Efficient Multiple Concatenated Codes With Turbo-Like Decoding for UEP Wireless Transmission of Scalable JPEG 2000 Images," in IEEE Access, vol. 7, pp. 6327-6336, 2019.

[12] L. Deng, et al., "Joint Coding and Adaptive Image Transmission Scheme Based on DP-LDPC Codes for IoT Scenarios," in IEEE Access, vol. 7, pp. 18437-18449, 2019.

[13] I. E. Bocharova, et al., "BP-LED Decoding Algorithm for LDPC Codes Over AWGN Channels," IEEE Transactions on Information Theory, vol. 65, no. 3, pp. 1677-1693, 2019.

[14] A. Matin, B. Dai, Y. Huang and X. Wang, "Ultrafast Imaging With Optical Encoding and Compressive Sensing," Journal of Lightwave Technology, vol. 37, no. 3, pp. 761-768, 2019.

[15] C. Shen and J. Yan, "High-Order Hadamard-Encoded Transmission for Tissue Background Suppression in Ultrasound Contrast Imaging: Memory Effect and Decoding Schemes," IEEE Transactions on Ultrasonics, Ferroelectrics, and Frequency Control, vol. 66, no. 1, pp. 26-37, 2019.

[16] X. He, L. Zhou, and J. Du, "PEG-Like Design of Binary QC-LDPC Codes Based on Detecting and Avoiding Generating Small Cycles," IEEE Transactions on Communications, vol. 66, no. 5, pp. 1845-1858, 2018.

[17] P. Gong, P. Song, and S. Chen, "Hadamard-Encoded Multipulses for Contrast-Enhanced Ultrasound Imaging," IEEE Transactions on Ultrasonics, Ferroelectrics, and Frequency Control, vol. 64, no. 11, pp. 1674-1683, 2017.

[18] P. Gong, P. Song, and S. Chen, "Delay-encoded harmonic imaging (DE-HI) in multiplane-wave compounding," in 2017 IEEE International Ultrasonics Symposium (IUS), Washington, DC, pp. 1-4, 2017.

[19] P. Gong, M. C. Kolios, and Y. Xu, "Delay-encoded transmission and image reconstruction method in synthetic transmit aperture imaging," IEEE Transactions on Ultrasonics, Ferroelectrics, and Frequency Control, vol. 62, no. 10 , pp. $1745-1756,2015$.

[20] P. Gong, P. Song, and S. Chen, "Ultrafast Synthetic Transmit Aperture Imaging Using Hadamard-Encoded Virtual Sources With Overlapping Sub-Apertures," IEEE Transactions on Medical Imaging, vol. 36, no. 6, pp. 1372-1381, 2017.

[21] J. Liu and J. Luo, "Compressed Sensing Based Synthetic Transmit Aperture for Phased Array Using Hadamard Encoded Diverging Wave Transmissions," IEEE Transactions on Ultrasonics, Ferroelectrics, and Frequency Control, vol. 65, no. 7, pp. 1141-1152, 2018.

[22] E. L. Villaverde, S. Robert, and C. Prada, "Ultrasonic Imaging in Highly Attenuating Materials With Hadamard Codes and the Decomposition of the Time Reversal Operator," IEEE Transactions on Ultrasonics, Ferroelectrics, and Frequency Control, vol. 64, no. 9, pp. 1336-1344, 2017.

[23] X. Song, et al., "Distributed Compressive Sensing for Cloud-Based Wireless Image Transmission," IEEE Transactions on Multimedia, vol. 19, no. 6, pp. 1351-1364, 2017.

[24] C. Wang, et al., "Efficient Compression of Encrypted Binary Images Using the Markov Random Field," IEEE Transactions on Information Forensics and Security, vol. 13, no. 5, pp. 1271-1285, 2018.

[25] Z. Chen, et al., "Efficient and Robust Image Coding and Transmission Based on Scrambled Block Compressive Sensing," IEEE Transactions on Multimedia, vol. 20, no. 7, pp. 1610-1621, 2018.

[26] W. Chen, "Modulating Phase via Rotation for Optical Encoding Based on Correlated Photon Imaging," IEEE Photonics Technology Letters, vol. 28, no. 4, pp. 540-543, 2016.

[27] C. Chen, et al., "Nonbinary LDPC Codes on Cages: Structural Property and Code Optimization," IEEE Transactions on Communications, vol. 63, no. 2, pp. 364-375, 2015.

[28] A. Khalid, et al., "Image transmission using unequal error protected multi-fold turbo codes over a two-user power-line binary adder channel,” IET Image Processing, vol. 9, no. 5, pp. 395-404, 2015.

[29] H. Yuan, et al., "Compressive sensing measurement matrix construction based on improved size compatible array LDPC code," IET Image Processing, 2015

[30] J. Zhang, H. Li, and C. W. Chen, "Distributed Lossless Coding Techniques for Hyperspectral Images," IEEE Journal of Selected Topics in Signal Processing, vol. 9, no. 6, pp. 977-989, 2015.

[31] A. Mahboub, et al., "An energy-efficient clustering protocol using fuzzy logic and network segmentation for heterogeneous WSN," International Journal of Electrical and Computer Engineering (IJECE), vol. 9, no. 5, pp. 4192-4203, 2019. 
[32] S. D. L. and K. Prasanna, "Energy efficient intelligent routing in WSN using dominant genetic algorithm," International Journal of Electrical and Computer Engineering (IJECE), vol. 10, no. 1, pp. 500-511, 2020.

[33] A. A. Hassan, et al., "Evaluate the performance of K-Means and the fuzzy C-Means algorithms to formation balanced clusters in wireless sensor networks," International Journal of Electrical and Computer Engineering (IJECE), vol. 10, no. 2, pp. 1515-1523, 2020.

[34] B. Gururaj and G. Sadashivappa, "Error-resilient transmission of image over challenging wireless communication channel," in AIP Conference Proceedings, vol. 2039, 2018.

\section{BIOGRAPHIES OF AUTHORS}

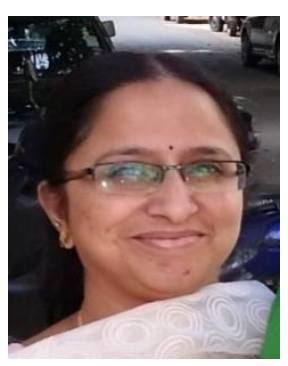

Bharathi Gururaj works as an assistant professor in the deparment of ECE, ACS College of Engineering, Bangalore, India. She has total of experience of 11 years in teaching. Her research are includes image processing and multimedia communications.

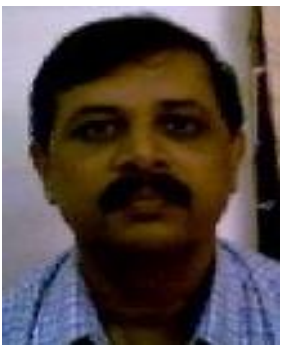

G. Sadashivappa is a professor in department of telecommunication engineering in RV College of Engineering, Bangalore, India. His has around 32 years of experience, in industry 2 years and research 3 years. His area of interest includesprocessing and applications and fiber optic communication. 